Montserrat Planelles Iváñez* UDK [811.133.1'373.4:355.01]:82"04/14"

Université d'Alicante

DOI: 10.4312/linguistica.58.1.9-21

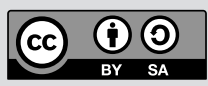

\title{
LES MOTS DE LA GUERRE AU MOYEN ÂGE : ÉTYMOLOGIE, USAGE ET ÉVOLUTION SÉMANTIQUE
}

\section{INTRODUCTION}

La guerre fait partie de l'univers socioculturel du Moyen Âge, que ce soient celles menées contre l'Angleterre ou les seigneurs féodaux, les croisades ou les batailles privées.

La guerre médiévale se manifeste comme à la fois un phénomène économique, comme une activité sportive, et plus généralement, ludique, comme une technique, et comme une libération de pulsions agressives. Cette multiplicité d'aspects explique l'abondance et la diversité du vocabulaire. (Matoré $1985: 156$ ).

Nous ne pouvons, donc, examiner celui-ci de manière exhaustive. En conséquence, notre analyse se limitera au vocabulaire de la guerre dans La Chanson de Roland ${ }^{1}$ et ne portera que sur des éléments significatifs autour de trois grands concepts : le guerrier, l'équipement et les armes et l'armée. Notre objectif est d'observer ce champ sémantique du point de vue lexicologique, avec une démarche diachronique. Nous présenterons les familles morphologiques et sémantiques des unités lexicales les plus représentatives en étudiant leur étymologie ainsi que leur évolution sémantique et leur survivance.

\section{LE GUERRIER}

Guerrier, guerrer. Nous trouvons dans le Trésor de la Langue française informatisé (désormais $T L F i$ ) la définition d'" homme qui fait la guerre » sous la forme guerrer, attestée en 1100 dans Roland v. 2066 : Li quens Rollant fut noble guerrer ${ }^{2}$. Le Dictionnaire du Moyen Français nous donne aussi l'acception, avec un pronom possessif, d'adversaire : Son guerrier « Son adversaire $»^{3}$.

Selon Matoré (1985 : 157), nous pouvons trouver les synonymes suivants de guerrier : guerreor, home de champ, escu « porteur d'un bouclier », étant ce dernier, selon le Dictionnaire du Moyen Français (désormais $D M F$ ), un usage métonymique signifiant «personne, chose qui protège et défend $»^{4}$. Le TLFi nous donne les synonymes

* montserrat.planelles@ua.es

1 L'édition que nous avons utilisée est celle de Joseph Bédier, La Chanson de Roland, 1920-1922, édition électronique, Édition du groupe « Ebooks libres et gratuits », 2004.

2 27/10/2016. http://www.cnrtl.fr/definition/guerrier/substantif

3 27/10/2016. http://www.cnrtl.fr/definition/dmf/guerrier

4 27/10/2016. http://www.cnrtl.fr/definition/dmf/\%C3\%A9cu?idf=dmfXhgYrmXart.revusYeXfe;str=0 
suivants : homme de troupe, homme d'infanterie : [Dans la hiérarchie milit.] Soldat qui est placé sous l'autorité d'un supérieur' ${ }^{5}$. Cette lexie se trouve dans le paradigme sémantique de « chevalier», avec soldoier, home d'armes, gens d'armes, josteor et fereor. De même, guerrier s'oppose à piéton, garçon et sergent.

Le paradigme morphologique de guerrier est très riche et vaste : aguerroyer, verbe, déguerroyer, verbe, enguerrer, verbe, enguerroyer, verbe, entreguerroyer, verbe, guerre, subst. fém., guerrer, verbe, guerreur, subst. masc., guerriable, adj., guerrier, subst. masc., guerrieux, adj., guerrir, verbe, guerroiement, subst. masc., guerroyable, adj., guerroyer, verbe, guerroyer, subst. masc., guerroyeresse, subst. fém., guerroyeur, subst.

Chevalier. Étymologie : du latin caballarium, dérivé de caballum, attesté à partir $\mathrm{du} \mathrm{V}^{\mathrm{e}}$ siècle, dénommant un soldat de la cavalerie, un aide militaire et au début du IX ${ }^{\mathrm{e}}$ siècle, un homme à cheval ou possédant un cheval. A partir du IX ${ }^{\mathrm{e}}$ siècle, il dénomme aussi un vassal, car le service des armes ne se conçoit que dans les relations vassaliques : le métier de combattant étant de plus en plus valorisé à l'époque (AndrieuxReix 1997 : 34).

Remarquons tout de même que les significations de chevalier ne sont pas les mêmes que celles de son étymon latin. Certes, avant 1180 , chevalier se limite à représenter le cavalier, le guerrier monté, avec un équipement spécifique, c'est-à-dire, un guerrier de métier. Cela signifie que le sens primitif de chevalier n'était attaché qu'à une fonction de service et de subordination jusqu'au $\mathrm{X}^{\mathrm{e}}$ siècle. Ce n'est qu'à partir du $\mathrm{XI}^{\mathrm{e}}$ siècle que l'on observe une valorisation du métier de combattant, source d'ascension sociale : « chevalier devient alors un terme laudatif, dénommant un guerrier d'élite, opposé à garçon, ribaut ou sergent » (Idem : 35). Ainsi, la nouvelle signification qui apparaît au XII ${ }^{\mathrm{e}}$ siècle devient dominante et parallèle à celle de chevalerie (voir infra). Ce dernier terme, qui concernait initialement un groupe de guerriers, a fini par dénommer une distinction sociale impliquant la noblesse et par conséquent la générosité, la bravoure et la protection au service de la société, c'est-à-dire, les valeurs qui seront appelées « chevaleresques » surtout à partir du XVIIe siècle, où chevalier désigne un membre de cette catégorie sociale.

Dès lors, la dénomination du cavalier était vacante : occupée par chevaucheur du $\mathrm{XIII}^{\mathrm{e}}$ au XVI $\mathrm{XI}^{\mathrm{e}}$ siècle, elle a été remplacée par cavalier à partir du XVI siècle.

En définitive, chevalier garde aussi la valeur d'une distinction honorifique, comme par exemple dans chevalier de la légion d'honneur et à partir du XVII siècle il correspond à un titre de noblesse inscrit dans une hiérarchie. Nous trouvons aussi des traces de l'idéologie « chevaleresque » assumée au XIII siècle dans les emplois déterminés de « chevalier de quelqu'un », où il signifie « protecteur ». Il faut remarquer que la relation avec « cheval » existant dans l'étymon caballarius s'efface dans chevalier. Les traits prédominants sont alors « militaire » et « service», qui ont rapproché chevalier de vassal. De même, le trait de «noblesse », fait que chevalier $^{6}$ entre en concurrence avec baron.

Chevalerie. Selon le $D M F$,

5 27/10/2016. http://www.cnrtl.fr/definition/homme/substantif

6 De vasselage fut asez chevaler, / Prozdom i out pur sun seignur aider (Roland, v. 25-26). Dans ces vers, chevaler a déjà les traits modernes de « militaire, service et noblesse » et peut concurrencer dans ce sens avec ber « baron» ou vassal. 
la chevalerie est une Institution militaire, d'un caractère religieux et héroïque, établie dans la noblesse féodale, exigeant de ceux qui y sont admis des qualités de loyauté et de vaillance, le mépris du danger, la protection des faibles, la défense de la foi, la courtoisie envers les femmes (DMF) ${ }^{7}$.

Le combat à cheval est donc l'action digne d'un chevalier considéré comme noble (Dunc avrez faite gente chevalerie, Roland v. 594). Le verbe chevalchier, de la même famille, est l'action jouée par le chevalier qui représente étymologiquement le guerrier qui possède un cheval de bataille ${ }^{8}$.

La famille morphologique de chevalier et chevalerie est vaste : cavalier, adj. et subst. masc., chevalerece, adj. fém., chevaleresse, subst. fém., chevalereusement, adv., chevalereux, adj., chevalerie, subst. fém., chevaleur, subst. masc., chevalier, subst. masc., chevalier de mer, subst. masc., chevalière, subst. fém.

Quant à cheval, provenant du latin caballum, il désigne l'animal en général, employé tant pour l'agriculture que pour la vie militaire. Le mot est passé en ancien français par le biais d'un emprunt aux populations celtiques de la Gaule cisalpine (Hélix 1999 : 46). Ce terme populaire et rustique a supplanté l'étymon noble latin equum, en raison d'une plus grande fréquence d'emploi et de la difficulté de prononcer un mot monosyllabique après la disparition de la syllabe finale $-u m(>* e q)$. En conséquence, dans l'usage, cheval désigne le plus souvent le cheval de bataille monté par le chevalier ${ }^{9}$. Le roncin, dont l'étymologie est incertaine, peut être considéré comme un synonyme de cheval dans son sens le plus général. C'est un cheval de labour, destiné aux paysans, mais aussi aux écuyers et aux bachelors, trop pauvres pour avoir un destrier. Cette lexie sera à l'origine de roussin. Nous trouvons aussi en ancien français le sommier, du bas latin sagma, signifiant « bât ». Celui-ci a donné son nom au coffre que l'on plaçait sur le dos du cheval, appelé some. Le sommier est devenu, donc, pas métonymie, un « cheval de charge », animal non noble, dont l'usage sert à humilier : c'est l'exemple de Ganelon dans La Chanson de Roland ${ }^{10}$.

Il est nommé aussi destrier ${ }^{11}$, terme dérivé de destre « main droite " ; dans ce cas, l'escuier (voir infra), le porteur de l'écu, mène le cheval par la main droite (sauf pendant le combat), car il ne fallait pas le fatiguer pour le conserver en pleine forme dans l'éventualité d'un combat (Matoré 1985 : 157 ; Hélix 1999 : 47).

7 27/10/2016. http://www.cnrtl.fr/definition/dmf/chevalerie?idf=dmfXdXrmXcbc;str=2

8 Guenes chevalchet suz une olive halte, / Asemblet s'est as sarrazins messag[es] (Roland, v. 266-367).

N'i perdrat Carles, li reis ki France tient, / Men escientre palefreid ne destrer, / Ne mul ne mule que deiet chevalcher, / Ne n'i perdrat ne runcin ne sumer, / Que as espees ne seit einz eslegiet (Roland, v. 755-759).

9 As porz d'Espaigne en est passet Rollant / Sur Veillantif, sun bun cheval curant (Roland, v. 11521153).

10 Ne mul ne mule que puissez chevalcher; / Getet serez sur un malvais sume (Roland, v. 480-481).

11 En Tachebrun, sun destrer est munted [Guenes li quens] (Roland, v. 347). 
Le cheval de bataille ou destrier s'oppose aussi au palefroi ${ }^{12}$, du bas latin paraveredus, étymon hétéroclite, car formé d'une préposition grecque - para et d'un mot francique latinisé veredus « cheval » : cheval d'une allure douce, destiné aux cérémonies mais aussi aux dames et demoiselles (Hélix 1999 : 47). Il s'oppose aussi au courtaud servant de monture aux pages et aux archers à cheval ainsi que pour l'usage ordinaire selon le $D M F^{13}$.

Escuier provient du bas latin scutarium, signifiant « soldat de la garde impériale qui portait un bouclier» ou escu, du latin scutum «bouclier». Nous trouvons par la suite en latin médiéval le mot scutarium avec le sens d'écuyer. L'escuier est d'habitude jusqu'au XII ${ }^{e}$ siècle un gentilhomme au service du chevalier et portant son écu. Dans ce sens, il est synonyme de sergent et de valet. A partir du XIII ${ }^{e}$ siècle, par contre, il désigne un jeune homme noble qui n'est pas encore armé chevalier. Il devient alors par extension un titre appliqué aux gentilshommes du dernier rang et puis, aux officiers d'un roi ou d'un prince ayant une fonction particulière : l'écuyer de cuisine, l'écuyer tranchant, le grand écuyer, étc. Aujourd'hui écuyer garde le sens de "professeur d'équitation », du XVII ${ }^{\mathrm{e}}$ siècle (Hélix 1999: 10).

La famille morphologique d'escuier est la suivante : écuiraille, subst. fém., écurie, subst. fém., écuyer, subst. masc., écuyeresse, subst. fém., écuyerie, subst. fém ${ }^{14}$.

Armez est le participe passé d'armer ${ }^{15}$, qui est utilisé avec le sens d' " hommes d'armes ». Les antonymes des armez sont la gent a pié, geude ou guilde " gens de pied, bande de soldats à pied $»^{16}$ ou pietaille " gens de pied, infanterie $»^{17}$ et la gent a cheval. De même, les valets d'armée sont nommés garçons ou rengaille, nom collectif.

La famille morphologique du verbe armer est remarquable : armable, adj., armatif, adj., armation, subst. fém., armée, subst. fém., armement, subst. masc., armer, verbe, armeret, subst. masc., armet, subst. masc., armeter, verbe, armette, subst. fém., armeur, subst. masc., désarmage, subst. masc., désarmer, verbe, enarme, subst. fém., enarmer, verbe, enarmes, subst. fém. plur., inarmé, adj., réarmer, verbe, renarmer, verbe ${ }^{18}$.

Garçon est issu du francique *wrakjo « vagabond ». Selon le $D M F^{19}$, ses acceptions sont les suivantes :

A. - « Domestique ; p. ext. homme vil et grossier».

1. «Valet (à l'armée, à la cuisine, à la chasse, ...) » - Garçon d'armes ; - Garçon de cuisine. « Marmiton »; - Garçon d'estables ; - Garçon à/de pied.

12 Puis que il sunt as chevals e as armes, Ja pur murir n'eschiverunt bataille (Roland v. 10951096), Vus n'i avrez palefreid ne destrer, Ne mul ne mule que puissez chevalcher (Roland, v. 479-480). Cheval qui sert pour le voyage, par opposition au destrier ou cheval de bataille. 5 novembre 2016. http://micmap.org/dicfro/search/glossaire-chanson-de-roland/palefroi

1327 octobre 2016. http://www.cnrtl.fr/definition/dmf/courtaud

145 novembre 2016. http://www.cnrtl.fr/definition/dmf/écuyer

15 Kar a mes oilz vi .IIII.C. milie armez (Roland, v. 682).

1627 octobre 2016. http://www.cnrtl.fr/definition/dmf/GUILDE

1727 octobre 2016. http://www.cnrtl.fr/definition/dmf/PIÉTAILLE

185 novembre 2016. http://www.cnrtl.fr/definition/dmf/armer

195 novembre 2016. http://www.cnrtl.fr/definition/dmf/garçon 
2. P. ext. péj. - « Homme au comportement vil et grossier, mauvais sujet »[Terme de mépris et d'injure]. En appellatif : - Faux garçon. « Traître »; - Mauvais garçon. « Malfaiteur, vaurien, lâche ».

B. - « Enfant de sexe masculin, jeune homme » - Vieux garçon. «Célibataire».

Sa signification en ancien français est donc double : d'un côté, « serviteur de statut social inférieur et occupant des fonctions subalternes ». Ses synonymes sont sergent et valet ; lorsqu'il s'occupe de l'entretien des chevaux, il est synonyme aussi d'escuier. D'un autre côté, il a une signification péjorative : «misérable, malotru, lâche ».

Quant à l'évolution de cette lexie, à partir du $\mathrm{XVI}^{\mathrm{e}}$ siècle, il perd sa signification médiévale et ne désigne qu'un " individu très jeune de sexe masculin » : sa connotation péjorative disparaît, même si le sème de domesticité se maintient jusqu'au XVII ${ }^{\mathrm{e}}$ siècle chez Molière. Actuellement les expressions garçon de café et garçon-coiffeur gardent encore le sème de service. Par contre, les traits de masculinité et de jeunesse l'emportent largement de nos jours. Remarquons à ce sujet que si garçon devient neutre quant à sa connotation péjorative, ce n'est pas le cas de valet, où domine aussi en ancien français le trait de jeunesse, mais il se spécialise ensuite au sens de " serviteur » et devient ainsi un terme péjoratif (Hélix 1999 : 8-9).

\section{L'ÉQUIPEMENT ET LES ARMES}

Garnement, qui signifie l'équipement et les armes, comprend l'aroi (dérivé du verbe areer " préparer ») et l'appareil « équipement», mais aussi l'armeüre « ensemble des armes défensives qui protègent le corps » et les armes ou herneis (> harnais). Revêtir son équipement de guerre se dit apareiller (Matoré 1985 : 158).

Nous n'allons traiter ici que armeüre et armes.

Le sens d'armeüre évolue au Moyen Âge. Selon le Dictionnaire Historique de la Langue française, armeüre > armure, apparaît tôt et désigne l'ensemble des armes défensives, poussant le mot arme, plus général, à ne plus désigner ces armes. L'ancien français connaît d'autres valeurs d'armeure, comme « homme en armes », «bataille », " exercice militaire », « escrime» et « armoiries » (Cfr. Aussi Godeffroy ${ }^{20}$ ). Certains de ces emplois, où armeüre était encore senti comme une sorte de dérivé collectif d'arme, étaient en concurrence avec armes. Armeüre, puis armure, s'est aussi employé pour «moyen de protection morale », par métaphore littéraire de sens concret. Dans ce contexte, nous allons décrire l'armure signifiant « armement; ensemble d'armes, défensives ou offensives ; pièce de cet ensemble » et en particulier, « ensemble des armes de défense qui protègent les combattants ; armure », même si ce mot signifie aussi « homme d'armes $»^{21}$.

L'armure ou armeüre se compose d'un haubert (osbert, halberc) ${ }^{22}$ - mot provenant du francique *halsberg (hals: «cou » et bergen « protéger »)- ou jazerenc

203 novembre 2016. http://micmap.org/dicfro/search/dictionnaire-godefroy/armeure

2128 octobre 2016. http://www.cnrtl.fr/definition/dmf/armure

22 Paien chevalchent par cez greignurs valees, / Halbercs vestuz e tres bien fermeez / Healmes lacez e ceintes lur espees, / Escuz as cols e lances adubees (Roland, v. 710-713). 
$>$ jaseran $^{23}$ " Tunique de mailles d'un chevalier (munie de manches, d'un gorgerin et d'une coiffe) " selon le DMF , de mailles ${ }^{24}$ laciées " chacun des petits anneaux formant le tissu métallique d'une armure; ce matériau fait de petits anneaux de fer engagés les uns dans les autres » selon de $D M F^{25}$, descendant jusqu'aux genoux et se terminant par un capuchon appelé coiffe "Partie de l'armure en contact direct avec la tête, sorte de capuchon porté sous le heaume et attaché au haubert $»^{26}$. Cette pièce a remplacé la brunie ou broigne, « cuirasse, corselet ${ }^{27}$ 《 justaucorps de cuir bardé d'anneaux de métal, mais ces deux termes coexistent jusqu'au XIII" siècle » (Matoré 1985 : 158). Voici la famille morphologique de broigne : brognée, subst. fém., brognon, subst. masc.

au même paradigme morphologique de haubert appartiennent les lexies suivantes : deshauberger, verbe, halzeran, subst. masc., haubergeon, subst. masc., haubergeonner, verbe, haubergeonnier, subst. masc., hauberger, verbe, haubergerie, subst. fém., haubergier1, subst. masc., haubergier 2 , verbe, haubert, subst. masc., hauboit, subst. masc.

Le haubert était placé sur une veste rembourrée appelée gambais $<$ wamba, « pourpoint rembourré qui se plaçait sous le haubert $»^{28}$. La tête est protégée par un helme $e^{29}<\mathrm{du}$ francique *helm $>$ heaume, « casque enveloppant la tete et le visage, muni d'ouvertures pour le visage $\aleph^{30}$ prolongé d'abord au-dessus du nez par un nasel ou nasal ${ }^{31}<$ nasus " partie du casque qui protégeait le nez »" (Matoré $1985: 158$ ). Le mot conserve au Moyen Âge son acception originelle et en acquiert une nouvelle dans le domaine de l'héraldique : " casque surmontant l'écu d'armes ». Les lexies bassinet et chapel de fer désignaient des casques plus légers et moins chers (Hélix 1999 : 23-24).

Bientôt le heaume s'allonge et recouvre le visage, avec des fentes pour la vue et pour la respiration, d'où le nom de ventail ou ventaille ${ }^{33}$ donné à cette couverture et par extension à l'ensemble du casque. « Dans les formes de casque les plus anciennes, elle consiste en un renfort percé de trous pour la respiration ; (...) la ventaille mobile est la partie inférieure de la visière ; elle forme avec le nasal une sorte de bec d'oiseau pointu propre à faire glisser les coups $\|^{34}$.

235 novembre 2016. http://www.cnrtl.fr/definition/dmf/JASERAN

24 Le blanc osberc, dunt la maile est menue (Roland, v. 1329).

253 novembre 2016. http://www.cnrtl.fr/definition/dmf/maille

263 novembre 2016. http://www.cnrtl.fr/definition/dmf/coiffe

273 novembre 2016. http://micmap.org/dicfro/search/dictionnaire-godefroy/broigne

283 novembre 2016. http://micmap.org/dicfro/previous/dictionnaire-godefroy/215/4/gambais

29 Tenez mun helme, unches meillor ne vi (Roland, v.629).

303 novembre 2016. http://www.cnrtl.fr/definition/dmf/heaume

31 Tut li detrenchet d'ici qu'al nasel (Roland, v. 1996), Jusqu'al nasel li ad frait e fendut (Roland, v. 3927).

322 novembre 2016. http://micmap.org/dicfro/previous/dictionnaire-godefroy/471/5/nasal

33 De sun osberc li rumpit la ventaille (Roland, v. 1293)

343 novembre 2016. http://micmap.org/dicfro/search/dictionnaire-godefroy/ventaille 
Aujourd'hui armeüre et toutes les dénominations de ses composants ne demeurent que dans le vocabulaire historique et n'ont rien à voir avec la réalité contemporaine de la guerre. En amont, le mot casque est privilégié face à heaume en français actuel.

Le chevalier porte aussi un escu pour parer les coups. Le mot provient du latin scutum " bouclier», issu du grec skutos " peau travaillé », " cuir ». Nous pouvons le trouver dans les expressions porter escu « combattre » ou rendre son escu « s'avouer vaincu ». Le paradigme morphologique de cette lexie est : écu, subst. masc., écuage, subst. masc., écucel, subst. masc., écué, adj., écusset, subst. masc., écusson, subst. masc., escuyer, subst. masc., scutifère, adj.

Escu peut signifier aussi " homme d'armes » au sens figuré par métonymie dès le milieu du XII siècle, et "protecteur » de la fin du XII ${ }^{e}$ au XIV ${ }^{e}$ siècle. Une nouvelle acception apparaît au XIII siècle dans le domaine de l'héraldique : « champ en forme de bouclier où sont représentées les pièces des armoiries » avant de désigner par métonymie les armoiries elles-mêmes au $\mathrm{XIV}^{\mathrm{e}}$ siècle. Le deuxième sens d'escu se développe en moyen français : il désigne une pièce d'or fin et puis une pièce d'argent. Actuellement il a été détrôné par l'euro mais il existe encore dans le domaine monétaire européen comme synonyme de bouclier (Hélix 1999 : 20-21). Nous trouvons l'expression escu boucler ${ }^{35}>$ bouclier, lorsqu'il sera garni d'une bocle. Un synonyme d'escu est targe ${ }^{36}$.

En ce qui concerne les armes, nous citerons l'espee ${ }^{37}$, la dague, le glaive; les armes de jet comme le javelot, l'arc ${ }^{38}$ et l'arbaleste ou arcbaleste ${ }^{39}$ ainsi que les instruments qui lancent : les saietes, fleches ou traits et les quarrels ${ }^{40}$.

D'une part, le mot espee provient du latin spatha. Cette arme était rangée dans un fourreau, d'où il fallait la sortir avant de combattre, d'où l'expression traire l'espee dans les récits médiévaux. D'autre part, l'étymologie de la dague est incertaine et il s'agit de l'arme des assassins et des mercenaires, par opposition à l'épee, propre aux chevaliers (Hélix 1999 : 18-19). Le glaive ou lance est une « épée ou espèce d'épée, arme tranchante, grand poignard $»^{41}$. L'étymon de glaive est gladius « épée », du latin; il en est de même pour lance, du latin lancea « arme de jet, pique », mot probablement d'origine celtique.

Le javelot est une espèce de lance : «Arme de jet en forme de lance courte $»^{42}$. L'arc est issu du latin arcus « arc » : c'était l'arme des corps d'archers grâce à sa légèreté et à son bas coût. Les projectiles de l'arc sont les flèches. Arbaleste provient d'arc. Il est issu du mot composé latin arcuballista, du latin arcus « arc » et ballista « machine de jet ». Les projectiles utilisés avec l'arbalète sont la saiete « flèche », le quarrel « flèche

35 Tanz [colps] ad pris sur sun escut bucler (Roland, v. 526).

36 Tute li freint la targe, ki est flurie (Roland, v. 3361).

37 Ceint Murglies, s'espee, a sun costed (Roland, v. 346).

38 «Dunez mei l'arc, que vos tenez el poign (Roland, v. 767).

39 D'un arcbaleste ne poet traire un quarrel (Roland, v. 2265).

40 D'un arcbaleste ne poet traire un quarrel (Roland, v. 2265).

415 novembre 2016. http://www.cnrtl.fr/definition/dmf/glaive

425 novembre 2016. http://www.cnrtl.fr/definition/dmf/javelot 
munie d'un fer pyramidal » et le vireton « flèche empenné en hélice avec des lamelles de bois ou de fer ». Les archers et les arbalétriers provenaient de la bourgeoisie (Hélix 1999 : 22-23).

Les vilains se servent de la maçuë > massue " pièce de bois à grosse tête noueuse (pouvant servir d'arme), massue $»^{43}$ et du gibet « bâton court avec une crosse, espèce de casse-tête $»^{44}$ ainsi que du flael > fleau « Arme de combat composée d'une masse de fer retenue par un bout de chaîne à l'extrémité d'une hampe " " fouet, baguette, verge ${ }^{45}$ et de la masse " arme formée d'un manche et d'une tête de métal (souvent garnie de pointes) » «bâton porté en signe d'autorité $»^{46}$. Remarquons que toutes ces « armes » sont très similaires.

Quant aux emblèmes militaires, nous trouvons des imprécisions : oriflamme ${ }^{47}$ et enseigne $^{48}$ (v. 3093, 3545). L'orie flambe signifie souvent l'enseigne royale, différente de la bane ou baniere royale parsemée de fleurs de lis. Le mot enseigne apparaît avec les sens de « indice » et de « cri de guerre » ainsi qu'avec celui de «banderole de l'extrémité d'une lance ». La baniere, insigne du droit féodal, sert de ralliement à ceux qui doivent à leur suzerain le service de l'ost ; nous trouvons aussi estandar $t^{49}$ (du francique *standhard « fixe ») : c'est la banderole qui se plante en terre et sert de point de ralliement aux combattants (Mattoré 1985 : 160). Et finalement, le gunfanun ${ }^{50}$ est un «étendard ou banderole à deux ou trois queues porté par les cavaliers sous le fer de la lance ; celui de Roland est blanc et les renges ${ }^{51}$ « franges » battent jusqu'aux mains du héros.

Comme dans toutes les sociétés guerrières, l'enseigne militaire est affectée d'un contenu symbolique. Sa forme et sa mobilité (elle flotte au vent) comme les couleurs et les attributs dont elle est pourvue font d'elle une promesse de victoire (v. 3267) ; elle est signe d'un commandement approuvé par Dieu : on comprend ainsi que son nom soit associé à un cri de guerre qui a un sens religieux : Montjoie (v. 1181) (Matoré 1985 : 161).

\section{L'ARMÉE}

$L^{\prime} o s t^{52}$ est un substantif qui désigne d'abord l'armée ennemie. Le substantif armée n'a été introduit qu'au $\mathrm{XIV}^{\mathrm{e}}$ siècle en concurrençant $o s t$, très couramment utilisé en ancien

435 novembre 2016. http://www.cnrtl.fr/definition/dmf/massue

445 novembre 2016. http://www.cnrtl.fr/definition/dmf/gibet

455 novembre 2016. http://www.cnrtl.fr/definition/dmf/FLÉAU

465 novembre 2016. http://www.cnrtl.fr/definition/dmf/masse

47 Gefreid d'Anjou portet l'orieflambe (Roland, v. 3093).

48 Geifreid d'Anjou ki l'enseigne teneit (Roland, v. 3545).

49 Dedavant sei fait porter sun dragon / E l'estandart Tervagan e Mahum/E un ymagene Apolin le felun (Roland, v. 3266-3268).

50 Laciet en su un gunfanun tut blanc (Roland, v. 1157).

51 Les renges li batent josqu'as mains (Roland, v. 1158).

52 Jo nen ai ost qui bataille li dunne (Roland, v. 18). 
français, bien qu'on lui substitue parfois esforz ${ }^{53}$ (v. 599), dérivé de forcier $>$ forcer ( $\mathrm{La}$ forcerie est la violence), et qui a aussi le sens moderne d' " effort ».

Conestablie et cumpaignie ${ }^{54}$ signifient " corps de troupe important ", synonymes aussi des lexies plus générales la gent, li noz et la rote, désignant la troupe en marche (Matoré 1985 : 161).

Le verbe semondre répond à l'activité de « mobilisation » et signifie « avertir ». Les barons semons, mandés ou ajustés sont ceux qui sont convoqués par leur suzerain pour le servise de l'ost. Les verbes querre ${ }^{55}$ et pourchacier $^{56}$ signifient « chercher » et « faire tous ses efforts ». La troupe est composée par des soldeiers ${ }^{57}$, qui sont des soldats mercenaires qu'il faudra soldre " payer» en leur versant une soldée.

$L^{\prime}$ eschiele ${ }^{58}$ représente la troupe en bataille et elle peut être commandée par le rei ${ }^{59}$, le conte, le conestable, le guion (cas sujet guis) « guide». L'activité du commandement est exprimée par de nombreux synonymes : aveir en bataille, governer, aveir en main. Commander ${ }^{60}$ a le sens général de « donner un ordre déterminé » ou de « confier».

Avant de se battre, les antagonistes essaient de parvenir à un accort $^{61}$ ou plait $^{62}$ (qui signifie aussi « traité »).

La concorde " accord entre les personnes, union, alliance, bonne entente $»^{63}$ peut être gradantée " effectuée » avec la parole finée " achevée ». Aliance conclue est synonyme d'amistié ${ }^{64}$ prise et assemblée faite. Cependant, il y a toujours des exigences de gages ou de réclamations des ostages $^{65}$, étant le sauf ostage la garantie d'un traité : le verbe ostagier signifie " promettre en donnant otages ».

Quand on ne parvient pas à faire acordement, quand aucun plait ou afaitement n'est possible, quand enfin on a semont l'ost, on desfie ${ }^{6 \sigma}$ "déclare les hostilités » et on

53 N'asemblereit jamais Carles si grant esfor (Roland, v. 599).

54 .XX. milie Francs unt en lur cumpaignie (Roland, v. 587).

55 Joes voell aler querre e entercer (Roland, v. 2180).

56 Li reis Marsilie s'en purcacet asez (Roland, v. 2612).

57 Ben en purrat luer ses soldeiers (Roland, v. 34).

58 La quinte eschele unt faite de Normans (Roland, v. 3045).

59 Carles li reis, nostre emperere magne (Roland, v. 1).

60 N'en parlez mais, se jo nel vos cumant (Roland, v. 273).

61 Se cest acorde ne vulez otrier, Pris e liez serez par poeste (Roland, v. 433-434).

62 Puis recevrat la lei que nus tenum, Ki ço vos lodet que cest plait degetuns (Roland, v. 225-226).

63 http://www.cnrtl.fr/definition/dmf/concorde (consulté le 2/11/2016).

64 Par amistiez, bel sire, la vos duin (Roland, v. 622).

65 Pur Pinabel en ostage renduz (Roland, v. 3950).

66 Jo desfiai Rollant le poigneor / E Oliver e tuiz lur cumpaignun; / Carles l'oïd e si nobilie baron. / Venget m'en sui, mais n'i ad traïsun (Roland, v. 3775-3779). 
meut $\left(\right.$ mouvoir $\left.^{67}\right)$ ou on fait la guerre ${ }^{68}$ : on guerreie $e^{69}$, la guerre sort (de sordre « jaillir »).

Les guerres au Moyen Âge sont des batailles rangées : les eschieles ${ }^{70}$ sont ordenées, conrées. Le conrei > conroi désigne l'armée rangée en ordre de combat mais il est aussi le synonyme bataille ${ }^{71}$ qui signifie « bataille » et « formation de combat », où les chevaliers sont disposés en plusieurs rangs en chargeant la lance.

Le front et la rereguarde ${ }^{72}$ forment l'armée. Il y a aussi le service de garde (du germanique *warda « surveillance ») (v. 192), qui est assuré par la gaite « veilleur » ou par l'eschargaite « troupe de guetteurs » et « sentinelle». Les espies « espions » apportent des enseignes « indices » précieuses. (Matoré $1985: 162$ ).

Le verbe attaquer est exprimé en ancien français par plusieurs verbes : asalir $^{73}>$ assaillir, envaïr ${ }^{74}$ (v. 2060), requerre, entreprendre. Dérivés d'envaïr sont : envaïssement et envaïe « attaque » « invasion », traduit à l'époque par le latinisme invasio et par surverse.

« On dresse une embuscade » correspond à l'époque médiévale à l'expression on enbusche un agait.

Le vocabulaire de la « bataille » est très important aussi. Ainsi, bataille ${ }^{75}$ « corps d'armée »; meslée $e^{76}$ 《lutte opiniâtre » ou « querelle », « combat singulier » (verbe mesler ${ }^{77}$ signifiant $«$ se prendre de querelle $\left.»\right) ;$ estor, estur ${ }^{78}$.

\section{CONCLUSION}

Même si le corpus analysé est restreint à trois grands concepts : le guerrier, l'équipement et les armes ainsi que l'armée, nous pouvons observer que le vocabulaire de la guerre au Moyen Âge est abondant et riche en synonymes et antonymes.

Les synonymes expriment non seulement l'importance du combat au Moyen Âge mais aussi l'imprécision du vocabulaire dans ce domaine : guerrier, guerreor, homme de champ, escu, homme de troupe, homme d'infanterie; cheval (générique), roncin, sommier ; cheval, destrier, cheval de bataille ; escuier, sergent, valet d'armée, garçon,

672 novembre 2016. « Mettre en mouvement une troupe » http://www.cnrtl.fr/definition/dmf/ mouvoir

68 Faites la guer[re] cum vos l'avez enprise (Roland, v. 210).

69 N'avrat talent, que ja mais vus guerreit (Roland, v. 579).

70 Icez eschieles ben les vunt ajustant (Roland, v. 3024).

71 Une bataille lur i rendent cil primes (Roland, v. 589).

72 Sa rereguarde avrat detres sei mise (Roland, v. 584); Qu'en rereguarde trover le poüsum (Roland, v. 624).

73 Se ne l'asaill, dunc ne faz jo que creire, / Si cunquerrai Durendal od la meie (Roland, v. 987-988).

74 Tut par seit fel ki nes vait envaïr (Roland, v. 2062).

75 Jo nen ai ost qui bataille li dunne (Roland, v. 18).

76 Dient paien: «Desfaimes la mellee » (Roland, v. 450).

77 Jo me crendreie, que vos vos meslisez (Roland, v. 257).

78 E Oliver chevalchet par l'estor (Roland, v. 1351). 
rengaille ; armez, hommes d'armes ; coiffe, brunie, broigne ; oriflamme, enseigne ; conestablie, cumpaignie ; aliance conclue, amistié prise, assemblée faite ; bataille, meslée, estor.

La polysémie s'avère être aussi une source d'imprécision : armeüre, escu, esforz.

Les antonymes montrent toujours des nuances de hiérarchie sociale : guerrier / piéton, garçon, sergent ; destrier ou cheval de bataille / palefroi / sommier, roncin, courtaud; chevalier / escuier, regent, valet ; armez / gent à pied, guilde, pietaille, gent à cheval; helme / bassinet, chapel de fer ; espée / dague; oriflamme / gunfanun / bane, banière royale.

La plupart des mots ont évolué du point de vue sémantique, comme chevalier et garçon, ou ont disparu, en ne subsistant que dans le domaine de l'histoire, comme les armes, pour des causes évidentes : la technique actuelle de l'armement est caractérisée par le progrès de la science el la guerre se fait autrement. Il en est de même pour les lexies faisant partie du paradigme morphologique, qui sont nombreuses dans tous les cas.

En ce qui concerne l'étymologie, les mots d'origine germanique sont nombreux dans ce champ sémantique, surtout dans le domaine de l'équipement, car ces éléments étaient méconnus pour les habitants de la Gaule et ce sont les peuples germaniques qui les ont introduits : heaume, haubert (*halsberg " ce qui protège »), coiffe (du haubert), hanste, gonfanon, baniere; gelde (germ. geld « troupe à pied»), estandart, etc. Cependant, il y a aussi beaucoup d'étymons latins, surtout dans le domaine des armes, déjà connues et utilisées par les Romains, comme par exemple espée, glaive, arc, arbaleste. La plupart des mots de l'armée sont aussi d'origine latine : ost, cumpaignie, semondre, querre, pourchacier, soldeier, eschiele, rei, amistié, etc

J'aurais voulu analyser aussi le vocabulaire des « actes de guerre » du Moyen Âge à nos jours mais l'espace est bref. J'ose cependant conclure que, hélas !, il continue d'être actuel, car les guerres sont toujours nombreuses et leurs causes se trouvent dans la nature humaine.

\section{Bibliographie}

ANDRIEUX-REIX, Nelly (1997) Ancien français. Fiches de vocabulaire. Paris : PUF. BÉDIER, Joseph (2004) La Chanson de Roland, 1920-1922, édition électronique. Édition du groupe « Ebooks libres et gratuits».

Dictionnaire du Moyen Français en ligne (DMF). http://www.cnrtl.fr/definition/dmf/

Dictionnaire Historique de la Langue française. Paris : Le Robert.

GODEFROY, Frédéric (1881) Dictionnaire de l'ancienne langue française et de tous ses dialectes du IX au XV siècle. http://micmap.org/dicfro/search/dictionnaire-godefroy/ MATORÉ, Georges (1985) Le vocabulaire et la société médiévale. Paris : Presses universitaires de France.

Trésor de la langue française informatisé (TLFi). http://atilf.atilf.fr/ http://www.cnrtl. fr/definition/ 


\section{Résumé \\ LES MOTS DE LA GUERRE AU MOYEN ÂGE : ÉTYMOLOGIE, USAGE ET ÉVOLUTION SÉMANTIQUE}

La guerre fait partie de l'univers socioculturel du Moyen Âge. Nous nous proposons de parcourir La Chanson de Roland pour étudier le lexique propre à la guerre. Notre analyse se limitera au vocabulaire de la guerre dans cette chanson de geste et ne portera que sur des éléments significatifs autour de trois grands concepts : le guerrier (guerrier, chevalier, cheval, escuier, armer), l'équipement et les armes (armeüre, haubert, escu, espée, javelot, oriflamme, enseigne, baniere) et l'armée (ost, armée, conestablie, cumpaignie, semondre, soldeiers, concorde, front, rereguarde, bataille, meslée). Notre objectif est d'observer ce champ sémantique du point de vue lexicologique, avec une démarche diachronique. En conséquence, nous présenterons les familles morphologiques et sémantiques des unités lexicales les plus représentatives en étudiant leur étymologie ainsi que leur évolution sémantique et leur survivance.

Mots-clés : lexicologie diachronique, étymologie, sémantique, guerre

\section{Abstract \\ WORDS OF WAR IN THE MIDDLE AGES: ETYMOLOGY, USAGE AND SEMANTIC EVOLUTION}

War is part of the socio-cultural universe of the Middle Ages. My objective is to go through La Chanson de Roland in order to study the lexicon of war. The analysis is limited to the vocabulary of war in this epic poem (chanson de geste) and focuses only on the significant elements, and more specifically on three major concepts: the warrior (guerrier, chevalier, cheval, escuier, armer), his equipment and weapons (armeüre, haubert, escu, espée, javelot, oriflamme, enseigne, baniere) and the army (ost, armée, conestablie, cumpaignie, semondre, soldeiers, concorde, front, rereguarde, bataille, meslée). The aim of this paper is to observe this semantic field from a lexical point of view by means of a diachronic approach. Consequently, the morphological and semantic families of the most representative lexical units are presented on the basis of their etymology and of their semantic evolution and survival.

Keywords: diachronic lexicology, etymology, semantics, war 


\section{Povzetek \\ VOJNO IZRAZJE V SREDNJEM VEKU: \\ ETIMOLOGIJA, RABA IN POMENSKI RAZVOJ}

Vojna je del družbeno-kulturnega sveta srednjega veka. V članku na podlagi $P e-$ smi o Rolandu analiziramo besedišče, značilno za vojno. Analiza, ki se omejuje na besedišče $\mathrm{v}$ tem junaškem epu, zadeva tri večje leksikalne koncepte: vojak (guerrier, chevalier, cheval, escuier, armer), oprema in orožje (armeüre, haubert, escu, espée, javelot, oriflamme, enseigne, baniere) ter vojska (ost, armée, conestablie, cumpaignie, semondre, soldeiers, concorde, front, rereguarde, bataille, meslée). Članek omenjena pomenska polja preučuje z diahronega leksikološkega vidika. Predstavljene so morfološke in pomenske družine najbolj zastopanih leksikalnih enot. Podana je tudi etimološka in pomensko-razvojna analiza izrazov.

Ključne besede: diahrono besedoslovje, etimologija, semantika, vojna 